\title{
Aneurisma de arteria renal: revisión de la literatura y reporte de caso
}

\section{Renal artery aneurysm: Literature review and case report}

\author{
Víctor A. Aguilar-Garay*, Wenceslao Fabián-Mijangos, Juan C. Moreno-Rojas, Damaso Hernández-López, \\ Teresa M. Casares-Bran y Guillermo Díaz Terán-Aguilera \\ Departamento de Angiología, Cirugía Vascular y Endovascular, Hospital Regional General Ignacio Zaragoza, Instituto de Seguridad y Servicios \\ Sociales de los Trabajadores del Estado, Ciudad de México, México
}

\section{Resumen}

\begin{abstract}
Antecedentes: El aneurisma de la arteria renal es una anormalidad vascular poco frecuente, la segunda en frecuencia de los aneurismas viscerales, se presenta en el $0.09 \%$ de la población general, la edad de presentación es a los 50 años, predominio en el sexo femenino, más frecuente del lado derecho. La localización más común es parenquimatosa de tipo sacular. La tasa anual de crecimiento es de 0,06-0,6 mm por año. Aunque son asintomáticos, el 70\% se asocia a hipertensión arterial como primera manifestación. Los factores que predisponen a la ruptura son considerados indicación absoluta para manejo quirúrgico convencional o endovascular. El manejo endovascular ha presentado una evolución constante, siendo una alternativa viable con una baja morbimortalidad. La embolización con material liquido o metálico o la exclusión con stent son opciones con demostrada efectividad, sin embargo, dependen de la disponibilidad de cada centro hospitalario. Objetivo: Presentamos el caso de una paciente hipertensa con el diagnóstico incidental de un aneurisma de la arteria renal derecha. Resultado: Se resolvió vía endovascular por contar con criterios de ruptura, presenta buena evolución posquirúrgica, se realiza seguimiento con ultrasonido renal sin evidencia de flujo en aneurisma.
\end{abstract}

Palabras clave: Aneurisma arteria renal. Endovascular. Exclusión. Embolización. Stent.

\section{Abstract}

Background: Renal artery aneurysm (RAA) is a rare vascular abnormality, the second in frequency of visceral aneurysms, it occurs in $0.09 \%$ of the general population, the age of presentation is 50 years, predominance in sex female, more frequent on the right side. The most common location is parenchymal saccular type. The annual growth rate is 0.06-0.6mm per year. Although they are asymptomatic, $70 \%$ are associated with arterial hypertension as the first manifestation. The factors that predispose to rupture are considered an absolute indication for conventional or endovascular surgical management. Endovascular management has shown a constant evolution, being a viable alternative with low morbidity and mortality. Embolization with liquid or metallic material or exclusion with a stent are options with proven effectiveness, however, they depend on the availability of each hospital center. Objective: We present a case of a hypertensive patient with the incidental diagnosis of an aneurysm of the right renal artery. Result: endovascular route was resolved due to rupture criteria, presents good postoperative evolution, renal ultrasound follow-up without evidence of aneurysm flow.

Key words: Renal artery aneurysm. Endovascular. Exclusion. Embolization. Stenting.

Correspondencia:

*Víctor A. Aguilar-Garay

E-mail: adrianaguilarrh@gmail.com

DOI: 10.24875/RMA.21000042
Disponible en internet: 29-11-2021 Rev Mex Angiol. 2021;49(4):133-138 www.RMAngiologia.com bajo la licencia CC BY-NC-ND license (http://creativecommons.org/licenses/by-nc-nd/4.0/). 


\section{Introducción}

La definición de aneurisma implica la dilatación de un segmento del vaso que duplica el diámetro de una arteria renal normal' ${ }^{1}$. El aneurisma de arteria renal (AAR) es una anormalidad vascular poco frecuente de la arteria que nutre al riñón, pero es potencialmente fatal.

La primera mención de un AAR fue por Rouppe en 1770, quien describió la muerte de un pescador que cayó sobre su flanco derecho y la autopsia revelo la ruptura de un pseudoaneurisma renal ${ }^{2}$. Aunque ya hace más de dos siglos que fue descrita, su baja incidencia no ha permitido adquirir suficiente experiencia sobre esta.

Según Urban, et al., el AAR es la segunda causa de aneurisma en las arterias viscerales (Tabla 1) ${ }^{3}$. La incidencia del $0.09 \%$ de la población general; la Universidad de Michigan reportó una incidencia del $0.1 \%$ en 8,500 arteriografías realizadas 4 .

La edad media de presentación de los AAR es a los 50 años, con predominio en pacientes del sexo femenino debido a la mayor prevalencia en pacientes con fibrodisplasia, con una relación mujer: hombre 5:15. El AAR secundario a una displasia fibromuscular frecuentemente es bilateral; cuando su presentación es unilateral afecta más comúnmente el lado derecho, en una relación de 2 a 1.

Las arterias renales surgen de la aorta a nivel del disco intervertebral entre L1 y L2. Existe gran variabilidad en la anatomía de la arteria renal y sus ramas, pero con mayor frecuencia la arteria renal principal se divide en una anterior y otra posterior. Las mediciones del diámetro de la arteria renal pueden variar dependiendo de la modalidad de imagen empleada. La media del diámetro de las arterias renales es $5.04 \pm 0.7 \mathrm{~mm}$. Las mediciones realizadas mediante angiografía han sido mayores $(5.68 \pm 1.19 \mathrm{~mm})^{6}$.

En cuanto a la etiología de los AAR, se encuentra que las causas son varias: enfermedad de Behçet, fibrodisplasia congénita, enfermedad de von Recklinghausen, aneurismas disecantes, aneurismas iatrogénicos y otras causas raras, como la mediólisis arterial segmentaria y vasculitis. En los pacientes ancianos, una de las causas más frecuentes de AAR es la enfermedad ateroesclerótica ${ }^{7}$.

En los aneurismas existe una debilidad de toda la pared mientras en los pseudoaneurismas hay una disrupción focal en una capa que causa la evaginación de un saco. En el trauma cerrado con desplazamiento anterior en riñones móviles, la rápida desaceleración
Tabla 1. Aneurismas de las arterias viscerales

\begin{tabular}{|l|c|}
\hline Arteria & Incidencia (\%) \\
\hline Arteria esplénica & $60-80$ \\
\hline Arteria renal & $15-20$ \\
\hline Arteria hepática & 20 \\
\hline Arteria mesentérica superior & 5.5 \\
\hline Arteria celíaca & 4 \\
\hline Arteria gástrica y gastroepiploica & 4 \\
\hline Arteria gastroduodenal y ramos \\
pancreáticos
\end{tabular}

Adaptada de Urban, et al., $2001^{13}$.

causa fractura de la íntima, predisponiendo a la disección subintimal con degeneración hacia aneurisma ${ }^{8}$.

Según Poutasse los AAR se clasifican en cuatro tipos ${ }^{9}$ :

- Tipo sacular: generalmente se presenta en la rama principal, cerca de la primera bifurcación y se asocia con fibrodisplasia media y aterosclerosis (Fig. 1A).

- Tipo fusiforme: se asocia a fibrodisplasia media principalmente y no presenta calcificaciones intrarrenales (Fig. 1B).

- Tipo disecante: afecta la rama principal y puede o no extenderse a las ramas secundarias, se subdivide de acuerdo con su origen en traumático, espontáneo y/o iatrogénico (Fig. 1C).

- Tipo intrarrenal: se asocia frecuentemente a arteritis (poliarteritis nodosa, granulomatosis de Wegener) y menos frecuentemente a ateroesclerosis, fibrodisplasia media, sífilis, trauma, malformaciones vasculares, tuberculosis o tumores. Derivan de las arterias interlobares y ramas periféricas (Fig. 1D).

La localización más frecuente es la extraparenquimatosa, aproximadamente el $85 \%$ de todos los $\mathrm{AAR}^{10}$. Los AAR saculares son los más comunes, constituyen entre el 60 y el $90 \%$, el $20 \%$ fusiformes y el $10 \%$ disecantes, se presentan como patología bilateral en el $20 \%$ de los casos y múltiples en $30 \%$. Se encuentran mayoritariamente en la bifurcación de la arteria renal ${ }^{11}$. La historia natural de los AAR no está clara. En series de casos se ha objetivado una tasa de crecimiento anual alrededor de 0.06-0.6 mm por año, sin diferencia en función de la morfología o el grado de calcificación de la formación aneurismática ${ }^{12}$. 


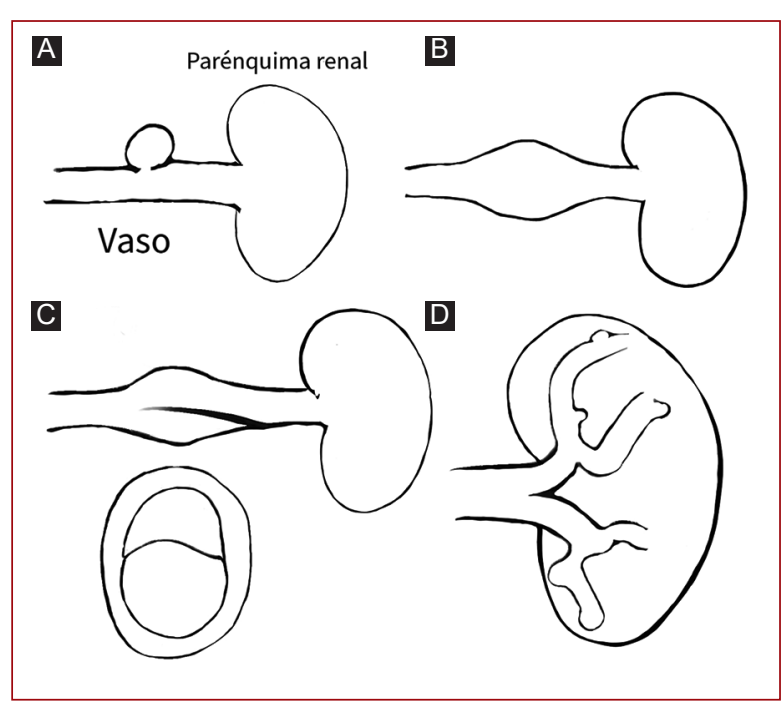

Figura 1. Clasificación de aneurisma renal según Poutasse.

La mayoría de los AAR son asintomáticos y se encuentran en estudios complementarios. Las manifestaciones clínicas más frecuentes son: hipertensión arterial (HTA) sistémica hasta el $70 \%$ de los casos (llegando al 100\%), debida a estenosis asociada, disección, formación de fístula arteriovenosa, tromboembolia, compresión de ramas vecinas por el propio aneurisma, dolor subcostal o en flanco, hematuria y excepcionalmente una masa palpable.

Una complicación rara pero grave es la rotura del aneurisma (3-5\%), que puede dar lugar a hematuria macroscópica si se rompe hacia la vía urinaria o a dolor en flanco e incluso choque hipovolémico si lo hace hacia el retroperitoneo $0^{13,14}$.

Los factores predisponentes para la ruptura son:

- Ausencia de calcificación o calcificación incompleta.

- Diámetro mayor de 2 centímetros.

- HTA asociada.

- Embarazo.

El embarazo está asociado a una mayor tasa de ruptura por el aumento del flujo sanguíneo renal, así como cambios hormonales que condicionan una mayor debilidad de la pared arterial por alteraciones del tejido elástico ${ }^{15}$, principalmente en el tercer trimestre del embarazo con una mortalidad materna alrededor del 50 al $70 \%$ y fetal del 80 al $100 \% 16,17$.

La tomografía es la modalidad diagnóstica contemporánea más frecuente, seguida por la resonancia magnética, la ultrasonografía y la arteriografía ${ }^{18}$.

Como consecuencia del aumento en el número de estudios diagnósticos con medio de contraste desde los años setenta, se ha incrementado el diagnóstico de esta patología, siendo hallazgos durante el estudio por otras patologías.

La evolución diagnóstica de los últimos años ha permitido ampliar el manejo terapéutico de AAR, ofreciendo un manejo quirúrgico convencional (abierto o laparoscópico) o endovascular, siempre individualizando cada caso para ofrecer el manejo óptimo.

El tratamiento quirúrgico está indicado en las siguientes situaciones ${ }^{19}$ :

- Aneurismas que producen isquemia renal.

- HTA secundaria al aneurisma.

- Aneurismas disecantes.

- Sintomatología local.

- AAR mayor de $2 \mathrm{~cm}$.

- Mujeres en edad fértil.

- Aumento progresivo de tamaño en los controles periódicos.

- Aneurisma con trombo detectable en angiografía y evidencia de embolización.

En cuanto a las técnicas quirúrgicas abiertas, puede realizarse una reparación in vivo (resección con anastomosis termino-terminal o colocación de prótesis) o ex vivo (cirugía de banco y autotrasplante renal).

Aunque en la actualidad existe consenso en la indicación de intervención en aneurismas sintomáticos, y sobre todo en mujeres embarazadas o en edad gestacional, el apropiado manejo de los aneurismas asintomáticos continúa indefinido. En los AAR que miden menos de $2 \mathrm{~cm}$ de diámetro y asintomáticos en pacientes normotensos, considerando su baja probabilidad de ruptura y su baja tasa de crecimiento, algunos autores consideran el manejo conservador ${ }^{20}$.

Con la aparición y el desarrollo de las técnicas endovasculares, se han limitado prácticamente a las siguientes indicaciones: a) aneurisma de la arteria renal después de la bifurcación, b) lesiones extensas del uréter, y c) cirugía conservadora en tumores renales en riñón único ${ }^{21}$. Con el apoyo de un estudio detallado de la vascularidad renal y la localización del aneurisma podemos determinar y elegir la mejor técnica endovascular que utilizar ${ }^{22}$.

Las estrategias endovasculares que permiten la preservación de la arteria renal mientras se logra con éxito la oclusión del aneurisma pueden realizarse mediante ${ }^{23}$ :

- Las embolizaciones con material líquido o metálico para lesiones distales y parenquimatosas.

- La exclusión con stent recubierto para aquellas lesiones proximales con afectación del tronco principal arterial o una de sus ramas. 


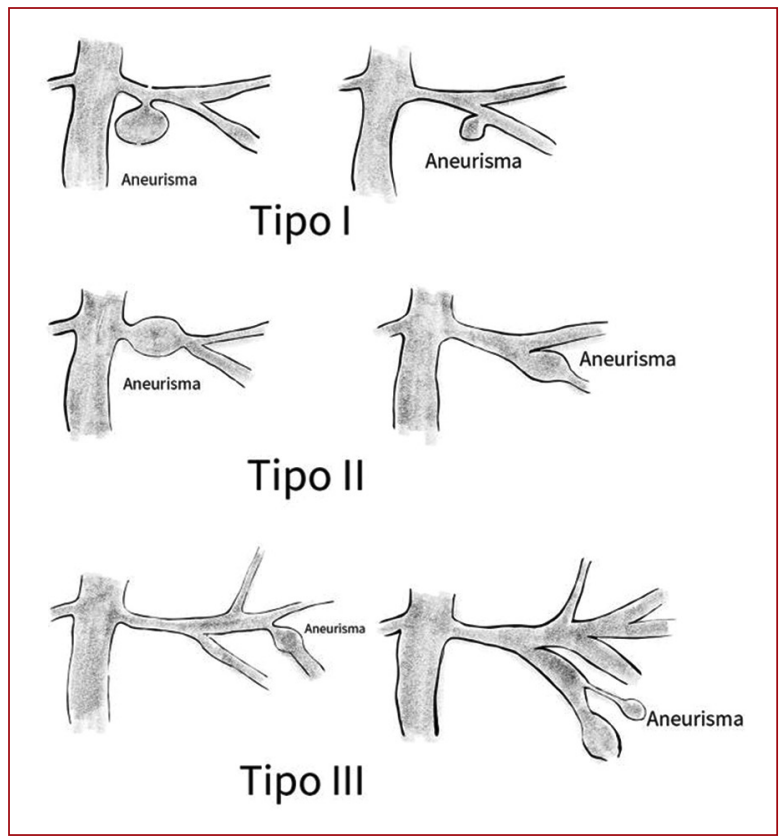

Figura 2. Clasificación angiográfica del aneurisma de la arteria renal, según Poutasse ${ }^{9}$.

Rundback, et al. propusieron un sistema de clasificación angiográfica ${ }^{24}$ (Fig. 2).

- Los AAR tipo I son saculares y emergen del tronco principal de la arteria renal o proximalmente desde una arteria segmentaria grande. Pueden ser tratados con éxito mediante stent o stent más embolización con coils.

- Los AAR de tipo II son fusiformes, asientan en la arteria renal principal y son mejor tratados quirúrgicamente.

- Los AAR tipo III se refieren a aneurismas intraparenquimatosos, afectando las pequeñas arterias segmentarias 0 accesorias, siendo mejor tratadas mediante embolización con coils con una pérdida mínima del parénquima ${ }^{25}$.

Cuando los aneurismas involucran los puntos de bifurcación de las arterias renales principales o segmentarias, el uso de endoprótesis puede resultar en la oclusión de algunas de las ramas. Se pueden sacrificar pequeñas ramas segmentarias, pero si los vasos de las ramas irrigan una porción significativa del parénquima renal, se pueden emplear técnicas alternativas.

Con la reparación endovascular del aneurisma se tiene que dar un seguimiento de la función renal, pues con el uso de esta técnica hay una disminución continua de la función renal en comparación con la reparación abierta, independientemente del nivel de fijación e

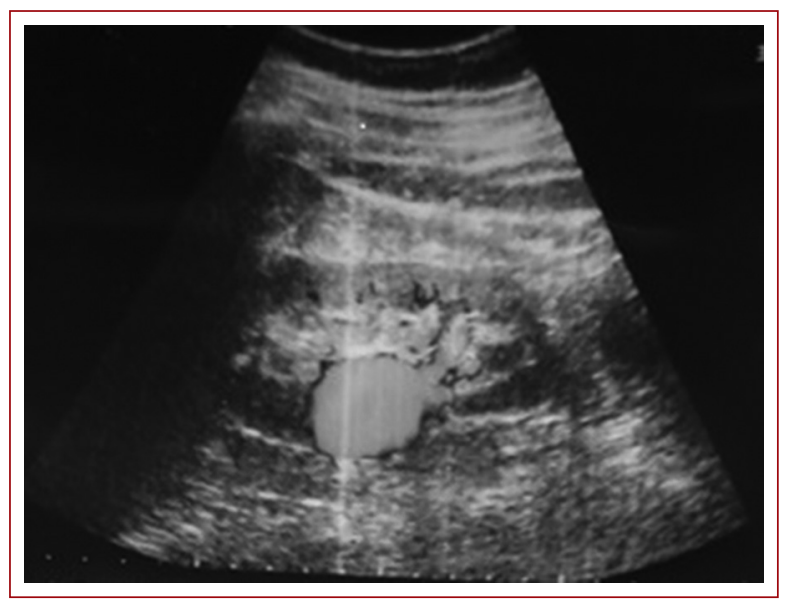

Figura 3. Ultrasonido Doppler abdominal, aneurisma de arteria renal derecha.

independientemente de la insuficiencia renal preexistente, y es necesario tomar en cuenta la tasa de filtración glomerular para la elección de la mejor técnica ${ }^{26}$.

Gonca (2019) reporta siete casos que se trataron con manejo endovascular en los que se logró un éxito clínico inmediato en pacientes tratados con embolización del saco primario hasta del $95 \%$. Con el uso de stent recubierto, la tasa de éxito clínico fue del $100 \%$ al año de seguimiento ${ }^{27}$.

Sin embargo, Buck, et al. reportan en su estudio la reparación de 6,234 aneurismas de arteria renal entre 1988 y 2011, comparando la cirugía abierta con el manejo endovascular con una mortalidad intrahospitalaria del $1.8 \%$ para la reparación endovascular y del $0.9 \%$ para reconstrucción abierta; la tasa de complicaciones fue del $12.4 \%$ para la reparación abierta frente al $10.5 \%$ para la reparación endovascular ${ }^{28}$.

\section{Objetivo}

El objetivo del este trabajo es presentar el manejo endovascular en el caso de un AAR como hallazgo incidental en una paciente hipertensa, con alto riesgo de ruptura.

\section{Caso clínico}

Mujer de 50 años de edad con el antecedente de HTA sistémica de cinco años de evolución en manejo con irbesartán con hidroclorotiazida.

Inicia su padecimiento ocho meses previos, con doIor abdominal difuso de intensidad $5 / 10$, realizándose un ultrasonido abdominal que evidencia imagen 


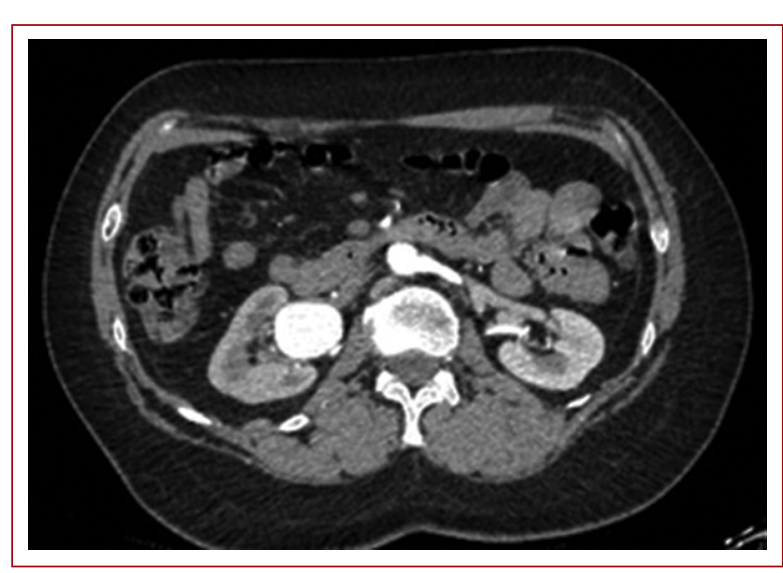

Figura 4. Tomografía axial computarizada, aneurisma de arteria renal derecha.

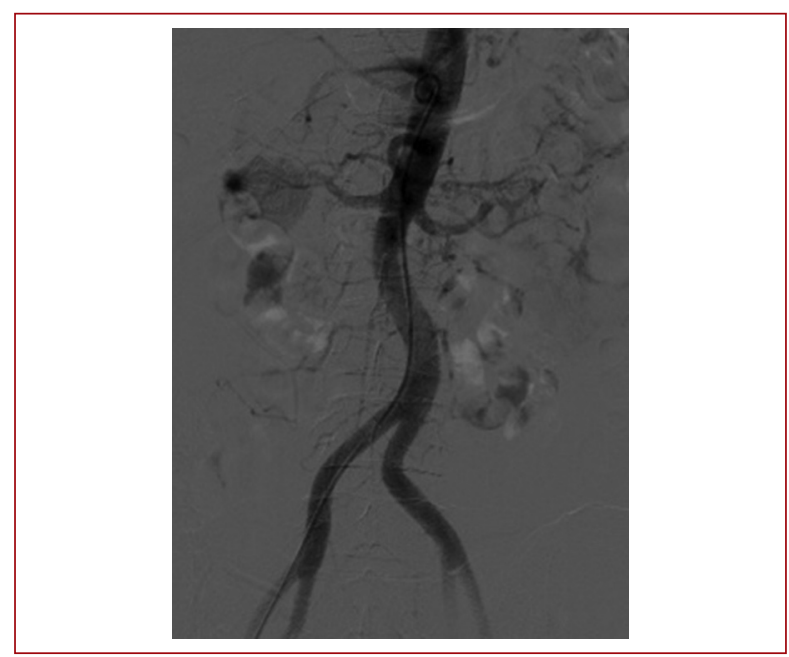

Figura 5. Arteriografía diagnóstica a través de catéter pigtail.

sacular vascularizada en la arteria renal derecha (Fig. 3). Biometría hemática con leucocitos de 6.5, neutrófilos 59\%, hemoglobina $14.9 \mathrm{mg} / \mathrm{dl}$, hematocrito 42.5 y plaquetas 244. Química sanguínea: glucosa $86 \mathrm{mg} / \mathrm{dl}$, creatinina 0.94 , Na 137, K 4.1 .

Completando el protocolo de estudio, se realiza una tomografía axial computarizada de abdomen simple y contrastada donde se observa la presencia de AAR derecha con un diámetro de 30.8 x 33.90 mm (Fig. 4).

Después del análisis del caso, lo clasificamos como un aneurisma tipo $\mathrm{A}$ de Poutasse. Teniendo múltiples opciones terapéuticas, se decide un manejo endovascular proyectando una exclusión de aneurisma + probable embolización con coils.

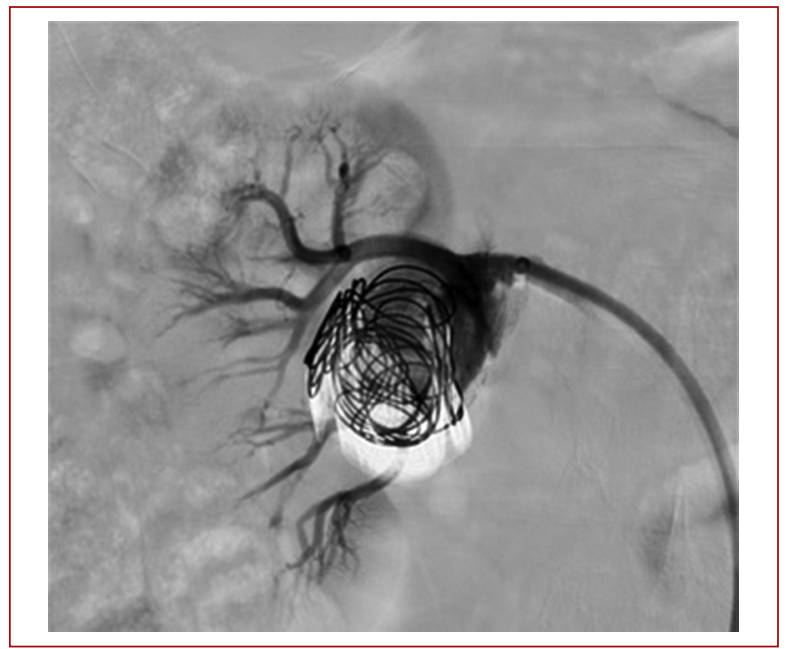

Figura 6. Angiografía arteria renal derecha. Aneurisma ya excluido con stent y embolizado con coils.

Se realiza en sala de hemodinámica arteriografía diagnóstica que evidencia el aneurisma, clasificándolo como tipo I de Rundback. Se realiza canulación selectiva de la arteria renal derecha, localizando el segmento distal de la arteria renal (Fig. 5).

Se identifica saco aneurismático en segmento extraparenquimatoso, por lo que se excluye con stent recubierto y se complementa con la liberación de coils (Fig. 6).

\section{Resultados}

Cursa con buena evolución posquirúrgica, con controles de laboratorio con creatinina en 1.0, urea en 16. Tolerando la vía oral y la deambulación, se decide su egreso a las $24 \mathrm{~h}$ posquirúrgicas. Se realiza un seguimiento a la semana y al mes con ultrasonido renal. Sin evidencia de flujo en aneurisma y función renal preservada.

\section{Conclusión}

Los AAR son poco frecuentes, las opciones terapéuticas son diversas, aunque la reparación quirúrgica en sus diferentes modalidades ya cuenta con una amplia experiencia y excelentes resultados, actualmente los procedimientos endovasculares han alcanzado un valor importante, pues desde el año 2000 el uso de estos métodos para la reparación de los aneurismas renales ha alcanzado resultados semejantes a la cirugía abierta, con una franca tendencia hacia ser superior. Dentro 
del manejo endovascular ha presentado apenas una mortalidad, morbilidad y estancia intrahospitalaria por debajo del manejo quirúrgico abierto. Sin embargo, habrá que considerar la disponibilidad de los materiales y la experiencia del cirujano endovascular.

\section{Financiamiento}

La presente investigación no ha recibido ninguna beca específica de agencias de los sectores públicos, comercial o con ánimo de lucro.

\section{Conflicto de intereses}

Los autores declaran no tener ningún conflicto de intereses.

\section{Responsabilidades éticas}

Protección de personas y animales. Los autores declaran que para esta investigación no se han realizado experimentos en seres humanos ni en animales.

Confidencialidad de los datos. Los autores declaran que han seguido los protocolos de su centro de trabajo sobre la publicación de datos de pacientes.

Derecho a la privacidad y consentimiento informado. Los autores han obtenido el consentimiento informado de los pacientes y/o sujetos referidos en el artículo. Este documento obra en poder del autor de correspondencia.

\section{Bibliografía}

1. Kitzing B, Vedelago J, Bajic N, Lai G, Waugh R. Stent-assisted coil embolization of a wide-necked renal artery aneurysm. J Radiol Case Rep. 2010;4:20-4.

2. Rouppe DL. Ingenti Anevrysmate spurio, dextro in latere abdominis, post lapsum. Nova Acta Phys-Med Acad Nat Curios. 1770. iv:67. Recuperado de: https://www.biodiversitylibrary.org/item/131767\#page/105/mode/1up.

3. Urban BA, Ratner LE, Fishman EK. Three-dimensional-volume-rendered CT angiography of the renal arteries and veins: Normal anatomy, variants, and clinical applications. Radiographics. 2001;21:373-86.
4. Klausner JQ, Lawrence PF, Harlander-Locke MP, Coleman DM, Stanley JC, Fujimura N; Vascular Low-Frequency Disease Consortium. The contemporary management of renal artery aneurysms. J Vasc Surg. 2015;61(4):978-84.

5. Romero O. Hipertensión arterial por aneurisma calcificado de la arteria renal. Primer caso nacional y revisión de la literature. Cir Cir. 2004;72:217-20.

6. Aytac S, Yigit Hasan H. Correlation between the diameter of the main renal artery and the presence of an accessory renal artery: Sonographic and angiographic evaluation. J Ultrasound Med. 2003;22:433-9.

7. Yoshioka T, Araki M, Ariyoshi Y, Wada K, Tanaka N, Nasu Y. Successful microscopic renal autotransplantation for left renal aneurysm associated with segmental arterial mediolysis. J Vasc Surg. 2017;66:261-4.

8. Iborra E. Aneurismas viscerales. Angiología. 2016;68(5):405-13.

9. Poutasse EF. Renal artery aneurysms. J Urol. 1975;113:443-9.

10. Bastounis E, Pikoulis E, Georgopoulos S, Alexiou D, Leppäniemi A, Boulafendis $D$. Surgery for renal artery aneurysms: a combined series of two large centers. Eur Urol. 1998;33(1):22-7.

11. López de la Franca $B$, Altés $P$, Pérez $P$, Sampere J, Lerma R, Llagostera $\mathrm{S}$. Tratamiento de un aneurisma intrarrenal mediante una embolización con coils: ¿es una buena alternativa para la hipertensión renovascular? Nefro Plus. 2016;8(2):179-82.

12. Klausner JQ, Harlander-Locke MP, Plotnik AN, Lehrman E, DeRubertis BG, Lawrence PF. Current treatment of renal artery aneurysms may be too aggressive. J Vasc Surg. 2014;59:1356-61.

13. Bonamigo TP, Erling N Jr, Faccini FP. Rupture of a secular renal artery aneurysm: report of a case. Surg Today. 2002;32(8):753-5.

14. Njinou Ngninkeu $B$, Eucher $P$, Vandenbossche $P$, Lacrosse $M$, van Cangh PJ, Lorge F. Ruptured aneurysm of the renal artery: a rara cause of macroscopic hematuria. Prog Urol. 2002;12(3):454-8.

15. Cura M, Elmerhi F, Bugnogne A, Palacios R, Suri R, Dalsaso T. Renal aneurysms and pseudoaneurysms. Clin Imaging. 2011;35:29-41.

16. Cohen JR, Shamash FS. Ruptured renal artery aneurysms during pregnancy. J Vasc Surg. 1986;6:51-9.

17. Henke PK, Cardneau JD, Welling TH $3^{\text {rd }}$, Upchurch GR Jr, Wakefield TW, Jacobs LA, et al. Renal artery aneurysms: a 35-year clinical experience with 252 aneurysms in 168 patients. Ann Surg. 2001;234:454-62; discussion: 462-3.

18. Nigro B, Ferrari J. Aneurisma de arteria renal: revisión. Revista Argentina de Cirugía Cardiovascular. 2019;XVII(3):100-7.

19. Hupp T, Allenverg JR, Post K, Roeren T, Meier M, Clorius JH. Renal artery aneurysm: surgical indications and results. Eur J Vasc Surg. 1992;6:477-86.

20. Brownstein AJ, Young E, Rajaee S, Yupeng L, Rizzo JA, Mojibian H, et al. Natural Historiy and management of renal artery aneurysms in a single tertiary referral center. J Vasc Surg. 2018;68:137-44.

21. Tran G, Ramaswamy K, Chi T, Meng M, Freise C, Stoller ML. Laparoscopic nephrectomy with autotransplantation: Safety, efficacy and longterm durability. J Urol. 2015;194:738-43.

22. Ferreira K. Techniques and strategies for the endovascular treatment of renal artery aneurysm. Rev Bras Cardiol Invasiva. 2014;22(1):64-7.

23. Orion KC, Abularrage CJ. Renal artery aneurysms: movement toward endo-vascular repair. Semin Vasc Surg. 2013;26:226-32.

24. Rundback JH, Rizvi A, Rozenblit GN, Poplausky M, Maddineni S, Crea $G$, et al. Percutaneous stent-graft management of renal artery aneurysms. J Vasc Interv Radiol. 2000;11:1189-93.

25. Li Z, Zhao Z, Qin F, Wei X, Sun Y, Liu J, et al. Outcomes of endovascular treatment and open repair for renal artery aneurysms: A single-center retrospective comparative analysis. J Vasc Interv Radiol. 2018;29(1):62-70.

26. Antonello M. Outcomes of endovascular aneurysm repair on renal function compared with open repair. Vasc Surg. 2013;58:886-93.

27. Gonca E, Erhan E, Bora P, Anil A, Ferhun B. Endovascular treatment of true renal artery aneurysms: a single center experience. Diagn Interv Radiol. 2019;25:62-70.

28. Buck DB, Curran T, McCallum JC, Darling J, Mamtani R, van Herwaarden JA, et al. Management and outcomes of isolated renal artery aneurysms in the endovascular era. J Vasc Surg. 2016;63(1):77-81. 